\title{
A Formalism for Expressing the Probability Density Functions of Interrelated Quantities
}

\author{
I. Lira ${ }^{1}$, D. Grientschnig ${ }^{2}$ \\ ${ }^{1}$ Department of Mechanical Engineering, Pontificia Universidad Católica de Chile, Vicuña Mackenna 4860, Santiago, Chile, \\ ilira@ing.puc.cl \\ ${ }^{2}$ Chemical Laboratories, Boehler Edelstahl, Mariazeller Str. 25, 8605 Kapfenberg, Austria, Dieter.Grientschnig@bohler- \\ edelstahl.at
}

\begin{abstract}
In this paper we address measurement problems involving several quantities that are interrelated by model equations. Available knowledge about some of these quantities is represented by probability density functions (PDFs), which are then propagated through the model in order to obtain the PDFs attributed to the quantities for which nothing is initially known. A formalism for analyzing such models is presented. It comprises the concept of a „base parameterization“, which is used in conjunction with the change-of-variables theorem. The calculation procedure that results from this formalism is described in very general terms. Guidance is given on how to employ it in practice by presenting both an elementary example and a much more involved one.
\end{abstract}

Keywords: Probability density functions, change of variables, parameterizations, Bayesian statistics.

\section{INTRODUCTION}

$\mathrm{I}^{\mathrm{N}} \mathrm{s}$ A PREVIOUS PAPER [1] we presented the main elements of a Bayesian procedure for assigning state of knowledge probability density functions (PDFs) to metrological quantities having essentially unique true values. In accordance with the prevalent view advocated in well-known international documents [2], [3], the mean and standard deviation of any such PDF are, respectively, taken as the best estimate and associated standard uncertainty of the quantity to which the distribution pertains, and the area under the PDF over a given interval is regarded as the probability of finding the true value of the quantity within that interval, given the available information.

For some of the quantities appearing in the model of measurement this information may be in the form of measurement data, for others some type of knowledge may be at hand (e.g., that their true values are known to be contained within prescribed intervals) and for still others there may be no information at all. The assignment of PDFs to those quantities about which something is known follows in general from tools such as Bayes' theorem [3, subclause 6.2] and the „Principle of Maximum Entropy“ [3, subclause 6.3], but expert's judgment is also needed, e.g., to identify discordant observations [4]. These PDFs are then propagated through the measurement model that links the corresponding quantities with those for which there is no knowledge. In this way, the PDFs for the latter quantities are obtained.

The procedure explained in [1] relies on the use of Dirac delta functions for effecting the propagation of probability distributions analytically [3, subclause 5.2]. Though the delta function is a very powerful concept, the rules for its manipulation may not be familiar to some metrologists. For this reason, in this paper we employ an alternative but entirely equivalent treatment based on the more widely known change-of-variables theorem [5]-[7]. To this end we provide a formal definition of the concept of „parameterization“, which is common in Bayesian statistics
[8],[9] but which in metrology has hitherto been used rather intuitively [10]. Two examples illustrate the application of the formalism.

\section{STATEMENT OF THE PROBLEM}

Consider a set of quantities $\mathbf{X}=\left\{X_{1}, \ldots, X_{m}\right\}$ interrelated through a consistent system of equations of the form

$$
F_{i}\left(\mathbf{X}_{i}\right)=0, \quad i=1, \ldots, n<m,
$$

where the $\mathbf{X}_{i}$ are subsets of $\mathbf{X}$, none of which is disjoint with all others, such that their union is equal to $\mathbf{X}$. It is assumed that none of the quantities is perfectly known and that their true values are unique. The goal is to obtain the PDF for the quantities of interest (the measurands) taking into account the existing information $I$ about some of the quantities in the system (1), which is hereafter symbolized by the letter $M$, for ,model“".

\section{ANALYSIS}

To analyze this problem it is useful to start from the concept of parameterization. In the present context, a parameterization is defined as a set $\mathbf{X}_{\mathbf{p}}=\left\{X_{\mathrm{p} 1}, \ldots, X_{\mathrm{p} l}\right\}$, where $l=m-n$, such that the model (1) allows all quantities in the complement of the parameterization to be expressed as explicit or implicit functions of some or all of the quantities it contains. There can be no more than $m ! /(n ! \times l !)$ parameterizations. For example, in the model $X_{1}=\sqrt{ } X_{2}$, $X_{3}=\ln X_{4}, X_{5}=X_{1} X_{3}$, the set $\left\{X_{1}, X_{3}\right\}$ is a parameterization but the set $\left\{X_{1}, X_{2}\right\}$ is not, because it is not possible to express $X_{3}, X_{4}$ and $X_{5}$ as functions of $X_{1}$ and $X_{2}$.

Three situations arise depending on the number of quantities to which the information $I$ refers. The first occurs when there exists no parameterization for which information on all its quantities is provided. It is then not possible to obtain the PDF for at least one of the quantities about which there is no knowledge. The proof is simple. Suppose that the 
available information refers to less than $l$ quantities and that it is precise, so that these quantities become known constants. The model (1) then turns into a „regular" underdetermined system of equations, from which no unique values of all unknowns can be found. This is true a fortiori if the information is imprecise (i.e., if for less than $l$ quantities only probability distributions rather than their exact values are given).

The second situation is frequently encountered in metrological practice. It occurs when there is information on just the $l$ quantities of a certain parameterization $\mathbf{X}_{\mathbf{b}}=\left\{X_{\mathrm{b} 1}\right.$, $\left.\ldots, X_{\mathrm{b} l}\right\}$, henceforth called the base parameterization, such that their joint PDF, denoted by $f_{\mathbf{b}}\left(\xi_{\mathbf{b}} \mid I\right)$, is either given or can be constructed by duly processing the available information (as explained, e.g., in section 3 of [1]). Here, the symbol $\xi_{\mathbf{b}}$ designates the set of variables that represent the possible values of the quantities $\mathbf{X}_{\mathbf{b}}$. In this case, the PDF for the quantities in any other parameterization $\mathbf{X}_{\mathbf{p}}$ can be obtained from the change-of-variables theorem

$$
f_{\mathbf{p}}\left(\xi_{\mathbf{p}} \mid I, M\right)=\left|J_{\mathbf{p b}}\right| \times f_{\mathbf{b}}\left[\mathbf{G}_{\mathbf{b}}\left(\boldsymbol{\xi}_{\mathbf{p}}\right) \mid I\right],
$$

where the $l$ functions $\mathbf{G}_{\mathbf{b}}$ are defined by

and

$$
X_{\mathrm{b} k}=G_{\mathrm{b} k}\left(\boldsymbol{X}_{\mathbf{p}}\right), \quad k=1, \ldots, l
$$

$$
J_{\mathbf{p b}}=\left|\frac{\partial \mathbf{G}_{\mathbf{b}}\left(\xi_{\mathbf{p}}\right)}{\partial \xi_{\mathbf{p}}}\right|
$$

is the determinant of the Jacobian matrix of the transformation. The symbols standing for ,given $I^{\prime}$ and "given $I$ and $M$ " in the arguments of the PDFs have been added for clarity; they may be dropped in practice, as in section 5 below. The functions $\mathbf{G}_{\mathbf{b}}$ (which need not be explicit) are obtained from the model (1); they are assumed to be differentiable at least once within the domains of interest of the quantities in the base parameterization. Equation (2) needs to be modified if the mapping $\mathbf{X}_{\mathbf{b}}=\mathbf{G}_{\mathbf{b}}\left(\mathbf{X}_{\mathrm{p}}\right)$ is not one-to-one in all quantities, but this brings about only a formal complication that is of no interest to our exposition. Note that the intersection of $\mathbf{X}_{\mathbf{b}}$ and $\mathbf{X}_{\mathbf{p}}$ may or may not be empty.

In this second situation the $l$ quantities forming the base parameterization are frequently called input quantities. Similarly, the $n$ quantities completely unknown originally are called output quantities, some or all of which may be the measurands. Their joint PDF may be obtained by including the output quantities in the above-mentioned parameterization $\mathbf{X}_{\mathbf{p}}$. Provided the latter also contains quantities that are not measurands, subsequent integration of the $\operatorname{PDF} f_{\mathbf{p}}\left(\xi_{\mathbf{p}} \mid I, M\right)$ over these quantities is required.

Finally, in the third situation information exists on more than $l$ quantities. It is easily seen that in this case one can use the change-of-variables theorem with different choices of $\mathbf{X}_{\mathbf{b}}$, leading to different PDFs $f_{\mathbf{p}}\left(\xi_{\mathbf{p}} \mid I, M\right)$ for any fixed parameterization $\mathbf{X}_{\mathbf{p}}$. This means that there is more than enough information. Either some of it must be discarded in order to keep just a single base parameterization (and consequently, a single PDF for any parameterization $\mathbf{X}_{\mathbf{p}}$ ) or some compromise must be reached if all information is to be retained. (The same may happen in case information is provided on all quantities of a set of size $l$ that is not a parameterization. For example, in the model $X_{1}=\sqrt{ } X_{2}$, $X_{3}=\ln X_{4}, X_{5}=X_{1} X_{3}$, providing information on both $X_{1}$ and $X_{2}$ would be more than enough with respect to these two quantities, but still not sufficient to establish the PDFs for $X_{3}, X_{4}$ and $X_{5}$. So this would, in fact, represent a situation of the first type.) The case of more than enough information is of interest in metrology, but it will not be addressed in the examples below.

\section{ONE OUTPUT QUANTITY}

The GUM [2] and its Supplement 1 [3] address explicit measurement models of the form

$$
X_{m}=F\left(\mathbf{X}_{\mathbf{b}}\right),
$$

where the single measurand $X_{m}$ is initially completely unknown and the information $I$ pertains only to the input quantities in the base parameterization $\mathbf{X}_{\mathbf{b}}=\left\{X_{1}, \ldots, X_{m-1}\right\}$. These models are easily handled with the present formalism. Indeed, by setting, e.g., $\mathbf{X}_{\mathbf{p}}=\left\{X_{1}, \ldots, X_{\mathrm{m}-2}, X_{m}\right\}$, (5) may be (partially) inverted according to

$$
\begin{aligned}
& X_{i}=G_{i}\left(X_{i}\right), \quad i=1, \ldots, m-2, \\
& X_{m-1}=G_{m-1}\left(X_{1}, \ldots, X_{m-2}, X_{m}\right) .
\end{aligned}
$$

Then (2) yields

$$
\mathrm{f}_{\mathrm{p}}\left(\xi_{\mathrm{p}} \mid \mathrm{I}, \mathrm{M}\right)=\left|\frac{\partial \mathrm{G}_{\mathrm{m}-1}}{\partial \xi_{\mathrm{m}}}\right| \times \mathrm{f}_{\mathrm{b}}\left(\xi_{1}, \ldots, \xi_{\mathrm{m}-2}, \mathrm{G}_{\mathrm{m}-1} \mid \mathrm{I}\right)
$$

Similar equations are obtained by choosing other parameterizations $\mathbf{X}_{\mathbf{p}}$ that contain $X_{m}$.

\section{A. Example}

As an example, suppose the model is

$$
X_{3}=\frac{X_{2}}{X_{1}}
$$

and let the base parameterization be $\mathbf{X}_{\mathbf{b}}=\left\{X_{1}, X_{2}\right\}$. We then have, with $\mathbf{X}_{\mathbf{p}}=\left\{X_{1}, X_{3}\right\}$,

$$
f_{1,3}\left(\xi_{1}, \xi_{3} \mid I, M\right)=\left|\xi_{1}\right| f_{1,2}\left(\xi_{1}, \xi_{1} \xi_{3} \mid I\right)
$$

or, with $\mathbf{X}_{\mathbf{p}}=\left\{X_{2}, X_{3}\right\}$,

$$
f_{2,3}\left(\xi_{2}, \xi_{3} \mid I, M\right)=\frac{\left|\xi_{2}\right|}{\xi_{3}^{2}} f_{1,2}\left(\frac{\xi_{2}}{\xi_{3}}, \xi_{2} \mid I\right) \text {. }
$$

The PDF for the measurand $X_{3}$ can now be obtained by marginalizing either of these two equations. Seemingly different but mathematically identical expressions for $f_{3}\left(\xi_{3} \mid I, M\right)$ are obtained. 
Incidentally, it is easily shown that by integrating (10) and (11) with respect to $\xi_{3}$ one gets $f_{1}\left(\xi_{1} \mid I, M\right)=f_{1}\left(\xi_{1} \mid I\right)$ and $f_{2}\left(\xi_{2} \mid I, M\right)=f_{2}\left(\xi_{2} \mid I\right)$, meaning that the model does not increase the state of knowledge about those quantities belonging to the base parameterization.

\section{B. Numerical illustration}

To illustrate, suppose (as in [10]) that $X_{3}$ is the length of a gauge block at a reference temperature $T_{0}$, that $X_{2}$ is its length at temperature $T$ and that $X_{1}$ is equal to $1+\alpha\left(T-T_{0}\right)$, where $\alpha$ is the linear thermal expansion coefficient. The information $I$ consists of knowing that $\alpha$ is contained within an interval of width $w$ centered at the value $a$, and that the mean of $N$ direct measurements of $X_{2}$ is $x_{2}$ with standard deviation $s_{2}$. For simplicity, assume that $T$ is measured with negligible uncertainty, so that it becomes perfectly known.

Since the information about $X_{1}$ is independent from that about $X_{2}$, the joint PDF for these two quantities is equal to the product of their individual PDFs, $f_{1}\left(\xi_{1} \mid I\right)$ and $f_{2}\left(\xi_{2} \mid I\right)$. The former is a rectangular distribution of width $w\left(T-T_{0}\right)$ centered at $1+a\left(T-T_{0}\right)$ [3, paragraph 6.4.2] while the latter may be taken as a $t$-distribution with location parameter $x_{2}$, scale parameter $s_{2} / \sqrt{ } N$ and $N-1$ degrees of freedom [3, paragraph 6.4.9]. Fig.1. shows the PDF for $X_{3}$ that results from the numerical integration of $f_{1,3}\left(\xi_{1}, \xi_{3} \mid I, M\right)$ over $\xi_{1}$, or of $f_{2,3}\left(\xi_{2}, \xi_{3} \mid I, M\right)$ over $\xi_{2}$, when $T-T_{0}=5 \mathrm{~K}, a=12 \times 10^{-6} \mathrm{~K}^{-1}$, $w=10^{-6} \mathrm{~K}^{-1}, N=5, x_{2}=50.0030 \mathrm{~mm}$ and $s_{2}=0.0016 \mathrm{~mm}$. The mean and standard deviation of this PDF are $x_{3}=50.0000 \mathrm{~mm}$ and $u_{3}=0.0010 \mathrm{~mm}$, respectively.

It is worth mentioning that the recommendation in [3] about the use of a $t$-distribution follows from Bayes' theorem and the assumption that the data are generated in accordance with a Gaussian random process of unknown mean and variance. The latter then becomes a further quantity that does not enter into any parameterization because it does not appear in the measurement model. This is in contrast to the concept of parameterization as used in [10].

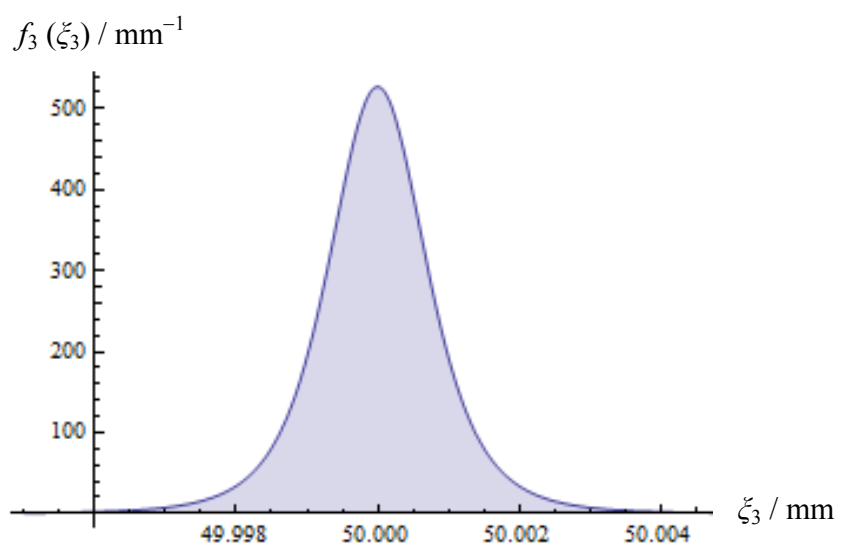

Fig.1. Probability density function for the length of a gauge block $X_{3}=X_{2} / X_{1}$ given information about $X_{2}$ and $X_{1}$.

\section{EXAMPLE: MEASUREMENT OF FLOW RATE}

An analytically more complicated example, but conceptually just as simple, is similar to the one considered in subsection 4.3 of [1]. The model consists of the DarcyWeisbach and Colebrook-White equations, which are commonly used for determining pipe-flow resistance in fully developed, steady state and incompressible flow within a pipe running full, see e.g., [11]. Consider a straight segment of a circular pipe of length $L$ and diameter $D$ inside which a fluid moves with average (bulk) velocity $V$. The pressure drop between the two ends of the segment is given by

$$
\Delta p=\lambda \frac{\rho V^{2} L}{2 D},
$$

where $\lambda$ is the dimensionless Darcy-Weisbach friction factor and $\rho$ is the density of the fluid. The friction factor depends on the flow regime and on the material of the pipe through its internal roughness $\varepsilon$. In case the Reynolds number $\operatorname{Re}=V D \rho / \mu$ is greater than about 4000 , where $\mu$ is the dynamic viscosity of the fluid, the flow is turbulent. One then obtains the friction factor from the (implicit) Colebrook-White equation

$$
\frac{1}{\sqrt{\lambda}}=-2 \log _{10}\left(\frac{2.51}{\operatorname{Re} \sqrt{\lambda}}+\frac{\varepsilon}{3.7 D}\right) .
$$

Normally, these two equations are used for establishing the power needed by the prime mover to overcome the pressure loss in the design stage of a piping system. In this application, if the properties of the fluid are regarded as perfectly known, the base parameterization is the set $\{V, D, \varepsilon\}$ and the measurand is the pressure drop along a unit length of pipe. But if the system is already in place, the same equations provide a means for measuring the volumetric flow rate $Q$ through the duct. Thus, the mass flow rate $\dot{m}=\rho Q$ can be obtained in an alternative manner to direct measurement, e.g., by a vortex mass flowmeter [12]. The volumetric flow rate is given by

$$
Q=V A,
$$

where $A=\pi D^{2} / 4$ is the pipe's cross-section. In this situation the base parameterization is the set $\{L, D, \Delta p, \varepsilon\}$.

Table 1. Equivalence of symbols for the quantities in the flow rate example.

\begin{tabular}{|c|c|}
\hline Quantity & New symbol \\
\hline$L$ & $X_{1}$ \\
\hline$D$ & $X_{2}$ \\
\hline$V$ & $X_{3}$ \\
\hline$\lambda$ & $X_{4}$ \\
\hline$\Delta p$ & $X_{5}$ \\
\hline$\varepsilon$ & $X_{6}$ \\
\hline$Q$ & $X_{7}$ \\
\hline
\end{tabular}


Let us analyze this second scenario where, for simplicity, the symbols for the relevant quantities are redefined as shown in Table 1. The model then becomes

$$
\begin{gathered}
X_{5}=a \frac{X_{1} X_{3}^{2} X_{4}}{X_{2}}, \\
\frac{1}{\sqrt{X_{4}}}=-2 \log _{10}\left(\frac{b}{X_{2} X_{3} \sqrt{X_{4}}}+\frac{X_{6}}{c X_{2}}\right),
\end{gathered}
$$

and

$$
X_{7}=d X_{2}^{2} X_{3}
$$

where $a=\rho / 2, b=2.51 \mu / \rho, c=3.7$ and $d=\pi / 4$. (The fluid's properties are regarded as perfectly known.)

Let the base parameterization be $\mathbf{X}_{\mathbf{b}}=\left\{X_{1}, X_{2}, X_{5}, X_{6}\right\}$. Several parameterizations can now be chosen, as long as they contain the measurand $X_{7}$. For example, choosing $\mathbf{X}_{\mathbf{p}}=$ $\left\{X_{1}, X_{2}, X_{4}, X_{7}\right\}$ gives

$$
f_{1,2,4,7}\left(\xi_{1}, \xi_{2}, \xi_{4}, \xi_{7}\right)=J \times f_{1,2,5,6}\left(\xi_{1}, \xi_{2}, G_{5}, G_{6}\right),
$$

where

$$
\begin{gathered}
G_{5}=\frac{a}{d^{2}} \frac{\xi_{1} \xi_{4} \xi_{7}^{2}}{\xi_{2}^{5}}, \\
G_{6}=c \xi_{2}\left(10^{-1 /\left(2 \sqrt{\xi_{4}}\right)}-\frac{b d \xi_{2}}{\sqrt{\xi_{4} \xi_{7}}}\right),
\end{gathered}
$$

and

$$
J=\left|\frac{\partial G_{5}}{\partial \xi_{4}} \frac{\partial G_{6}}{\partial \xi_{7}}-\frac{\partial G_{5}}{\partial \xi_{7}} \frac{\partial G_{6}}{\partial \xi_{4}}\right|=\frac{a c \ln 10}{2 d^{2}} \frac{\xi_{1} \xi_{7}}{\xi_{2}^{4} \sqrt{\xi_{4}}} 10^{-1 /\left(2 \sqrt{\xi_{4}}\right)}
$$

\section{A. Available information}

Suppose estimates $x_{2}$ for the diameter and $x_{6}$ for the roughness of a pipe are available, with associated standard uncertainties $u_{2}$ and $u_{6}$, respectively. Inside the pipe flows a liquid whose density and viscosity are known with negligible uncertainty. The pressure drop along a straight part of the duct is measured, giving the estimate $x_{5}$ with standard uncertainty $u_{5}$. Finally, the distance $x_{1}$ between the pressure gauges is also measured with standard uncertainty $u_{1}$. All these estimates are obtained independently of one another. According to the „Principle of Maximum Entropy“, it is then reasonable to assign univariate Gaussian PDFs to $X_{1}, X_{2}, X_{5}$ and $X_{6}$. We restrict the calculation to the case where the ratios $x_{i} / u_{i}$ are reasonably large, say greater than three. This enables us to formally extend the support of all four quantities to the whole real line [13]. We then have

$$
f_{1,2,4,7}\left(\xi_{1}, \xi_{2}, \xi_{4}, \xi_{7}\right)=J \times f_{1}\left(\xi_{1}\right) f_{2}\left(\xi_{2}\right) f_{5}\left(G_{5}\right) f_{6}\left(G_{6}\right),
$$

where

$$
f_{i}\left(\xi_{i}\right)=\frac{1}{\sqrt{2 \pi} u_{i}} \exp \left(-\frac{1}{2} \frac{\left(\xi_{i}-x_{i}\right)^{2}}{u_{i}^{2}}\right), \quad i=1,2,
$$

$$
f_{i}\left(G_{i}\right)=\frac{1}{\sqrt{2 \pi} u_{i}} \exp \left(-\frac{1}{2} \frac{\left(G_{i}-x_{i}\right)^{2}}{u_{i}^{2}}\right), \quad i=5,6
$$

and where $J, G_{5}$ and $G_{6}$ are as above.

In these circumstances, integration over $\xi_{1}$ can be performed analytically. The result is

$$
f_{2,4,7}\left(\xi_{2}, \xi_{4}, \xi_{7}\right)=H \times K \times f_{2}\left(\xi_{2}\right) f_{6}\left(G_{6}\right)
$$

where

$$
\begin{gathered}
H=\frac{a c \ln 10}{2 d^{2}} \times \frac{\xi_{7} 10^{-1 /\left(2 \sqrt{\xi_{4}}\right)}}{\xi_{2}^{4} \sqrt{\xi_{4}}}, \\
K=\exp \left(-\frac{1}{2} \frac{\left(k x_{1}-x_{5}\right)^{2}}{k^{2} u_{1}^{2}+u_{5}^{2}}\right) \times \frac{u_{5}^{2} x_{1}+k u_{1}^{2} x_{5}}{\sqrt{2 \pi}\left(k^{2} u_{1}^{2}+u_{5}^{2}\right)^{3 / 2}},
\end{gathered}
$$

and

$$
k=\frac{a}{d^{2}} \frac{\xi_{4} \xi_{7}^{2}}{\xi_{2}^{5}} .
$$

However, the integrations over $\xi_{2}$ and $\xi_{4}$ to obtain the marginal $\mathrm{PDF} f_{7}\left(\xi_{7}\right)$ for the measurand $X_{7}$ have to be carried out numerically. If desired, the PDF $f_{4}\left(\xi_{4}\right)$ for the friction factor $X_{4}$ can be obtained likewise.

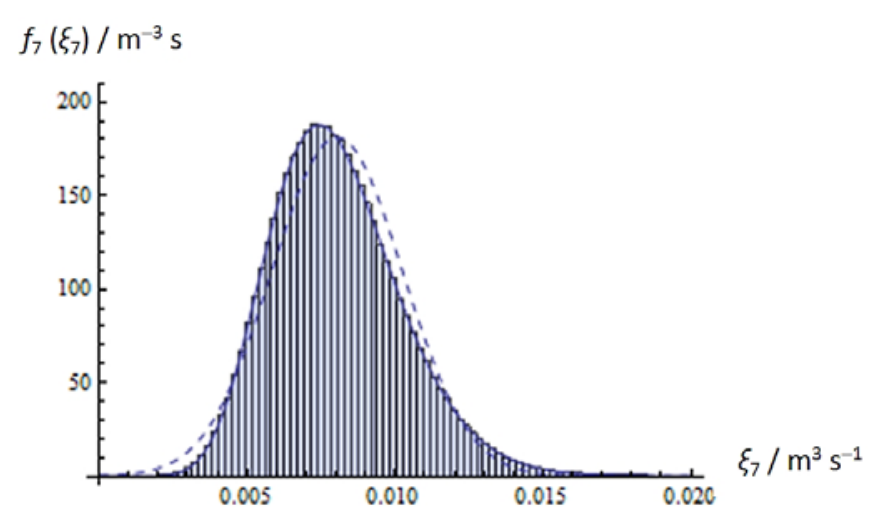

Fig.2. The solid line is the PDF for the volumetric flow rate $X_{7}$ of water in a pipe. The histogram is its numerical approximation computed by Monte Carlo integration. The dashed line is a Gaussian PDF with the same mean and standard deviation.

\section{B. Results}

As an illustration, suppose the fluid is water at $22{ }^{\circ} \mathrm{C}$, for which $\rho=998 \mathrm{~kg} / \mathrm{m}^{3}$ and $\mu=959 \times 10^{-6} \mathrm{~Pa} \mathrm{~s}$, flowing inside a pipe of diameter $x_{2}=10 \mathrm{~cm}$ and roughness $x_{6}=150 \mu \mathrm{m}$. The pressure drop over the distance $x_{1}=10 \mathrm{~m}$ is found to be $x_{5}=1180 \mathrm{~Pa}$. Assume that the associated standard uncertainties are equal to one tenth of the corresponding estimates. The PDF $f_{7}\left(\xi_{7}\right)$ is depicted by the solid line in Fig.2. Its mean is $x_{7}=0.0080 \mathrm{~m}^{3} / \mathrm{s}$ and its standard deviation is $u_{7}=0.0022 \mathrm{~m}^{3} / \mathrm{s}$. Its skewness reflects the strong nonlinearity of the model (15)-(17). For comparison, the dashed line in Fig.2. shows a Gaussian PDF with the same mean and standard deviation. 
The estimate $x_{7}$ allows calculating the velocity as $x_{3}=4 x_{7} /\left(\pi x_{2}{ }^{2}\right)=1.02 \mathrm{~m} / \mathrm{s}$, giving a Reynolds number greater than $10^{5}$. Therefore, the flow is fully turbulent, so the use of (16) is justified.

\section{Integration by Monte Carlo}

The Monte Carlo integration method proposed in Supplements 1 and 2 to the GUM [3],[14] can also be employed for finding a numerical approximation to $f_{7}\left(\xi_{7}\right)$. This method is entirely equivalent to applying the changeof-variables theorem. It is to be preferred, however, in instances that are too difficult to be addressed by analytical or numerical integration, e.g., if the number of variables is too large. In the present case the Monte Carlo method proceeds by sampling values $\xi_{1}, \xi_{2}, \xi_{5}$ and $\xi_{6}$ from the corresponding PDFs. The values

$$
\xi_{4}=\left[-2 \log _{10}\left(\frac{b \sqrt{a \xi_{1}}}{\xi_{2} \sqrt{\xi_{2} \xi_{5}}}+\frac{\xi_{6}}{c \xi_{2}}\right)\right]^{-2},
$$

$$
\xi_{3}=\left(\frac{\xi_{2} \xi_{5}}{a \xi_{1} \xi_{4}}\right)^{1 / 2},
$$

and

$$
\xi_{7}=d \xi_{2}^{2} \xi_{3}
$$

are then computed and the process is repeated a large number of times. The relative frequency of the $\xi_{7}$ values obtained after one million repetitions is shown by the histogram in Fig.2., confirming that it is an excellent numerical approximation to the $\operatorname{PDF} f_{7}\left(\xi_{7}\right)$.

One advantage of the Monte Carlo method is that the numerical approximations to the PDFs for $X_{3}$ and $X_{4}$ are immediately available as a by-product. They allow computing the means, standard deviations and correlation coefficients shown in Table 2.

Table 2. Means, standard deviations and (dimensionless) correlation coefficients computed by Monte Carlo integration.

\begin{tabular}{|l|c|l|l|c|c|c|}
\hline Quantity & Symbol & Mean & \multirow{2}{*}{$\begin{array}{c}\text { Standard } \\
\text { deviation }\end{array}$} & \multicolumn{3}{|c|}{ Correlation coefficients } \\
\cline { 5 - 7 } & & & $X_{3}$ & $X_{4}$ & $X_{7}$ \\
\hline Velocity $/ \mathrm{m} \mathrm{s}^{-1}$ & $X_{3}$ & 1.01 & 0.10 & 1.0 & -0.71 & 0.83 \\
\hline Friction factor & $X_{4}$ & 0.02371 & 0.00088 & -0.71 & 1.0 & -0.84 \\
\hline Flow rate $/ \mathrm{m}^{3} \mathrm{~s}^{-1}$ & $X_{7}$ & 0.008 & 0.0022 & 0.83 & -0.84 & 1.0 \\
\hline
\end{tabular}

\section{CONCLUSION}

A formalism for expressing the PDFs for interrelated quantities has been presented in general terms. The aim has been to identify clearly the different situations that may arise and to provide guidance by way of examples on how to employ the technique in practice. It relies on the concept of „base parameterization“, defined as a set of quantities constituting a parameterization for which information is provided so that its joint PDF can be constructed. The size of this set is equal to the total number of quantities minus the number of equations through which they are related. If information on a greater number of quantities exists, the problem cannot be solved uniquely. In case there is information on fewer quantities, one remains ignorant about at least one of the quantities that were initially completely unknown.

\section{REFERENCES}

[1] Lira, I., Grientschnig, D. (2010). Bayesian assessment of uncertainty in metrology: A tutorial. Metrologia, 47, R1-R14.

[2] BIPM, IEC, IFCC, ILAC, ISO, IUPAC, IUPAP, OIML (2008). Evaluation of measurement data Guide to the Expression of Uncertainty in Measurement. JCGM 100:2008 (GUM 1995 with minor corrections).
[3] BIPM, IEC, IFCC, ILAC, ISO, IUPAC, IUPAP, OIML (2008). Evaluation of measurement data Supplement 1 to the 'Guide to the expression of uncertainty in measurement' - Propagation of distributions using a Monte Carlo method. JCGM 101:2008.

[4] Barbato, G., Genta, G., Germak, A., Levi, R., Vicario G. (2012). Treatment of experimental data with discordant observations: Issues in empirical identification of distribution. Measurement Science Review, 12, 133-140.

[5] Kaplan, W. (1984). Advanced Calculus (3 ${ }^{\text {rd }}$ ed.). Reading, MA: Addison-Wesley.

[6] Jeffreys, H., Jeffreys, B.S. (1988). Methods of Mathematical Physics ( $3^{\text {rd }}$ ed.). Cambridge, England: Cambridge University Press.

[7] Possolo, A., Toman, B., Estler, T. (2009). Contribution to a conversation about the Supplement 1 to the GUM. Metrologia, 46, L1-L7.

[8] Gelman, A. (2004). Parameterization and Bayesian modeling. Journal of the American Statistical Association, 99, 537-545.

[9] Zwickl, D.J., Holder, M.T. (2004). Model parameterization, prior distributions, and the general time-reversible model in Bayesian phylogenetics. Systematic Biology, 53, 877-888. 
[10] Elster, C., Toman, B. (2009). Bayesian uncertainty analysis under prior ignorance of the measurand versus analysis using the Supplement 1 to the Guide: A comparison. Metrologia, 46, 261-266.

[11] Benedict, R.P. (1980). Fundamentals of Pipe Flow. New York: John Wiley.

[12] Li, Z., Sun, Z. (2013). Development of the vortex mass flowmeter with wall pressure measurement. Measurement Science Review, 13, 20-24.
[13] Lira, I. (2009). The probability distribution of a quantity with given mean and variance. Metrologia, 46, L27-L28.

[14] BIPM, IEC, IFCC, ILAC, ISO, IUPAC, IUPAP, OIML (2011). Evaluation of measurement data Supplement 2 to the 'Guide to the expression of uncertainty in measurement' - Extension to any number of output quantities. JCGM 102:2011.

Received June 13, 2012. Accepted April 4, 2013. 